Document downloaded from:

http://hdl.handle.net/10251/60325

This paper must be cited as:

Avsarkisov, V.; Hoyas Calvo, S.; Oberlack, M.; García Galache, JP. (2014). Turbulent plane Couette flow at moderately high Reynolds number. Journal of Fluid Mechanics. 751:1-10. doi:10.1017/jfm.2014.323.

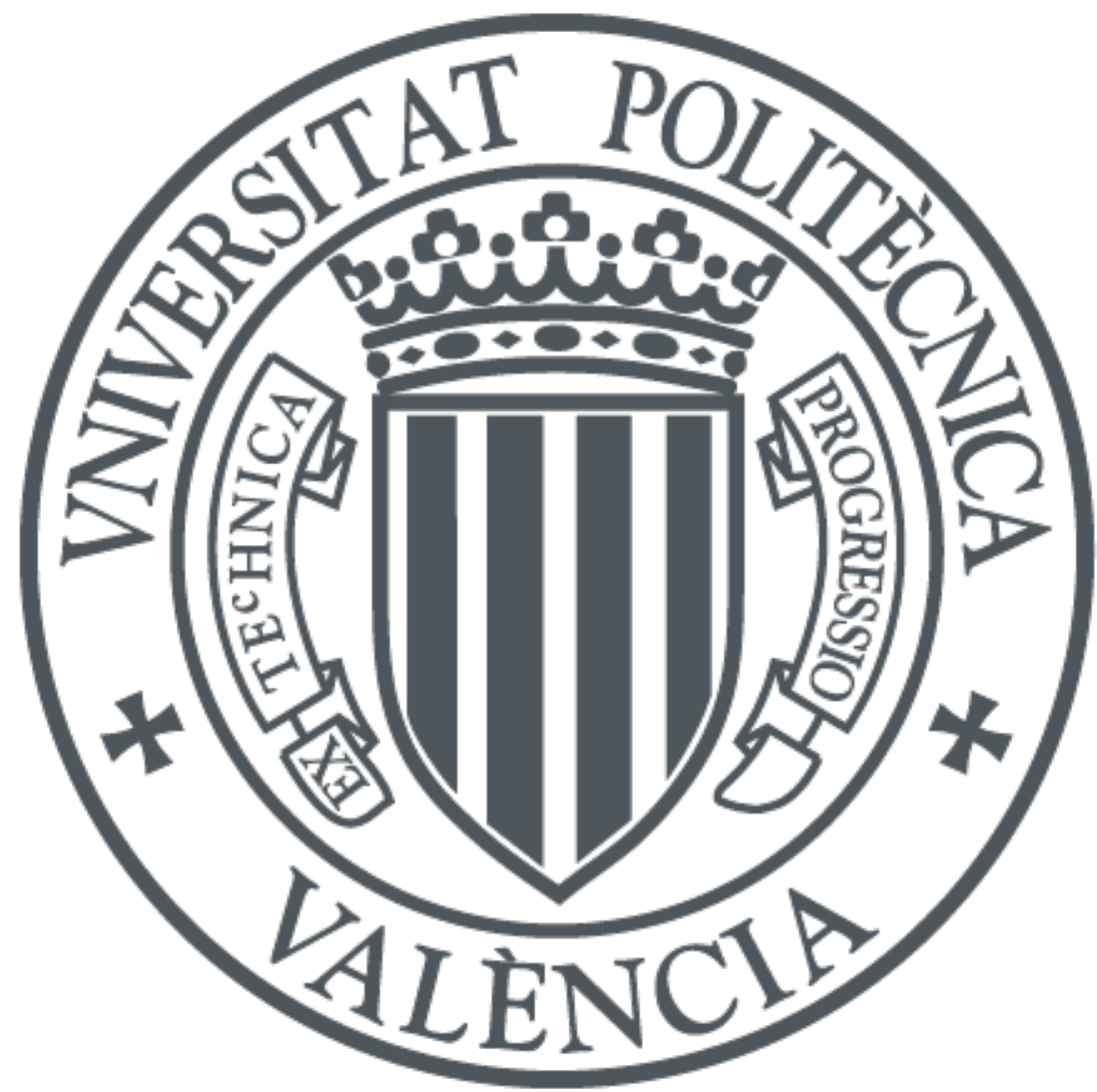

The final publication is available at

http://dx.doi.org/10.1017/jfm.2014.323

Copyright Cambridge University Press (CUP): STM Journals

Additional Information 


\title{
Turbulent Plane Couette flow at moderately high Reynolds number
}

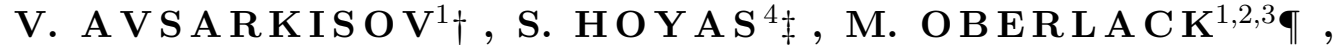 \\ AND J.P. G. GA L A CHE \\ ${ }^{1}$ Chair of Fluid Dynamics, TU Darmstadt, Otto-Berndt-Str. 2, 64287 Darmstadt, Germany \\ ${ }^{2}$ Center of Smart Interfaces, TU Darmstadt, Alarich-Weiss-Str. 10, 64287 Darmstadt, Germany \\ ${ }^{3}$ GS Computational Engineering, TU Darmstadt, Dolivostr. 15, 64293 Darmstadt, Germany \\ ${ }^{4}$ CMT Motores Térmicos, Univ. Politècnica de València, València, Spain
}

(Received ?; revised ?; accepted ?. - To be entered by editorial office)

A new set of numerical simulations of turbulent plane Couette flow in a large box of dimension $(20 \pi h, 2 h, 6 \pi h)$ at $R e_{\tau}=125,180,250$ and 550 is described and compared with simulations at lower Reynolds numbers, Poiseuille flows, and experiments. The simulations present a logarithmic near-wall layer and presently used to verify and revise previously known results. It was confirmed that fluctuation intensities in the streamwise and spanwise directions do not scale well in wall units. The scaling failure happens both near and away from the wall. On the contrary, the wall-normal intensity scales in inner units in the near-wall region and in outer units in the core region. The spectral ridge found in Hoyas \& Jiménez [Scaling of the velocity fluctuations in turbulent channels up to $R e_{\tau}=2003$, Physics of Fluids, 011702] for the turbulent Poiseuille flow can be also seen in the present flow. Away from the wall, very large scale motions are found spanning through all the length of the channel. The statistics of these simulations can be downloaded from the webpage of the Chair of Fluid Dynamics.

\section{Introduction}

Direct numerical simulation (DNS) has been a fundamental tool for the study of wall turbulence since the seminal work of Kim et al. (1987). For a detailed reference on the state of the art on this fascinating topic, see for instance the recent work of Jiménez (2013). Focusing on channel flow geometry and in particular on Poiseuille flows, moderate Reynolds numbers up to $R e_{\tau}=2003$ have been reached (Hoyas \& Jimenez 2006), but considerably less attention has been paid to plane Couette flows. The main issues are the long and wide structures existing in turbulent Couette flow, as it has been stated experimentally (Tillmark 1995, Kitoh et al. 2005; Kitoh \& Umeki 2008) and numerically

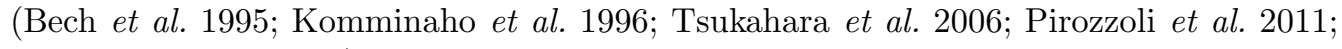
Bernardini et al. 2013). The necessity of large boxes to capture these structures makes the study of this flow much more computationally expensive than turbulent Poiseuille flow.

In this work, a new set of DNSs of a plane turbulent Couette flow has been performed within a computational box of $L_{x}=20 \pi h, L_{y}=2 h$ and $L_{z}=6 \pi h$, with spanwise and streamwise periodicities and at Reynolds numbers of $R e_{\tau}=125,180,250$ and 550, based on the friction velocity $u_{\tau}$ and on the channel half-width $h$. The simulations for the low $R e$ numbers are thus comparable to other, most recent simulations made by

$\dagger$ Email address for correspondence: v.avsarkisov@astro-ge.org

\pm Email address for correspondence: serhocal@mot.upv.es

ๆ Email address for correspondence: oberlack@fdy.tu-darmstadt.de 
Bernardini et al. (2013). Tsukahara et al. (2006) and the Poiseuille flow at $R e_{\tau}=550$ of Jiménez's group (del Álamo \& Jiménez 2003, Jimenez \& Hoyas 2008). Due to the well known box size issues that are related to the largest structures which form in the core region of the Couette flow it is important to mention that Bernardini et al. (2013) performed DNS at $R e_{\tau}=167$ in a box $(12 \pi h \times 2 h \times 4 \pi h)$, while Tsukahara et al. (2006) performed DNS at $R e_{\tau}=126$ in several boxes up to $(40 \pi h \times 2 h \times 4 \pi h)$. The method employed to obtain the flow evolution is similar to the one used by Kim et al. (1987) and Hoyas \& Jimenez (2006). The streamwise, wall-normal, spanwise coordinates are $x, y$, and $z$ and the corresponding velocity components are $u, v$ and $w$. The governing equations of the system are transformed into an equation for wall-normal vorticity $\omega_{y}$ and for the Laplacian of the wall-normal velocity $\phi=\nabla^{2} v$. The spatial discretization uses dealiased Fourier expansions in $x$ and $z$, and seven-point compact finite differences in $y$, with fourth-order consistency and extended spectral-like resolution (Lele 1992). The temporal discretization is a third-order semi-implicit Runge-Kutta scheme (Spalart 1991). Initial fields were taken from previously calculated Poiseuille flows, imposing the new boundary conditions. The code was validated with the results of Tsukahara et al. (2006), i.e. we performed simulation with exactly same parameters in similar box. Due to the simplicity of the obtained verification results they are not included in the present paper. Table 1 summarizes the parameters of the present simulations. The wall-normal grid spacing is adjusted to keep the resolution, $\Delta_{y}=1.5 \eta$, approximately constant in terms of the local isotropic Kolmogorov scale $\eta=\left(\nu^{3} / \epsilon\right)^{1 / 4}$ for the $R e_{\tau}=550$ case. In wall units, $\Delta y^{+}$varies from 0.92 at the wall up to $\Delta y^{+} \simeq 5.9$ at the centerline. In order to facilitate the comparison of structures in outer variables we used the same grid in wall-normal direction for all $R e_{\tau}=180-550$ cases. The running times are given both in terms of turnover periods for eddies of size $h$ and of velocity $u_{\tau}$, and flow-troughs. For the present box the former are approximately 2.5 bigger than the latter. Transitions until statistically steady state is reached are no contemplated in Table 1 . One of the tools used to asses that the statistically steady state has been reached is to compute the total shear stress, which should be one in Couette flows (See figure 1b). In the present study we restrict ourselves to describe the new data coming from these simulations and compare them with the results obtained for the pure Poiseuille flows. For a complete description of transition from Couette to Poiseuille flow the reader is referred to the work of Pirozzoli et al. (2011).

\section{Statistics}

The mean velocity profiles are shown in figure $1 \mathrm{a}$ scaled in outer and inner units. The new profiles agree well with the simulations of Tsukahara et al. (2006) and Bernardini et al. (2013) at $R e_{\tau}=125-250$. In figure 1 a the $\log$ law $U^{+}=1 / \kappa \log \left(y^{+}\right)+B$ has been plotted for $\kappa=0.41$ and $B=5.1$. Unlike the results of Kitoh et al. (2005) and Tsukahara et al. (2006) we do not see any dependency of $B$ on the friction Reynolds number in the range $R e_{\tau}=125-550$. This may be an indication that the Reynolds numbers at which the flow was studied in the past was not sufficiently high to investigate the near-wall log-law.

In order to analyze the inverse of the Kármán constant the log-indicator function $y \partial_{y} U^{+}$has been plotted in figures $2 \mathrm{a}$ and $2 \mathrm{~b}$ in outer and inner variables respectively. All curves, including the Poiseuille flow at $R e_{\tau}=550$ of del Álamo \& Jiménez $(2003)$, collapse well up to their first minimum, at $y^{+} \simeq 60$. As was suggested in Hoyas \& Jimenez (2006) this value can be taken as a lower limit for the logarithmic layer. After this point, Couette and Poiseuille flows differ significantly. It appears that the curve in the log- 


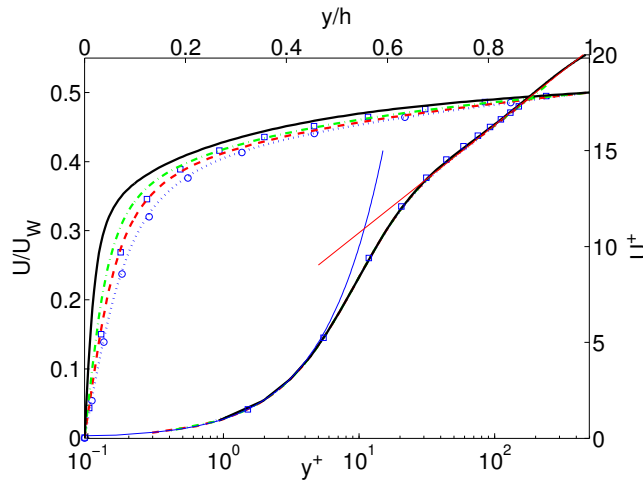

(a)

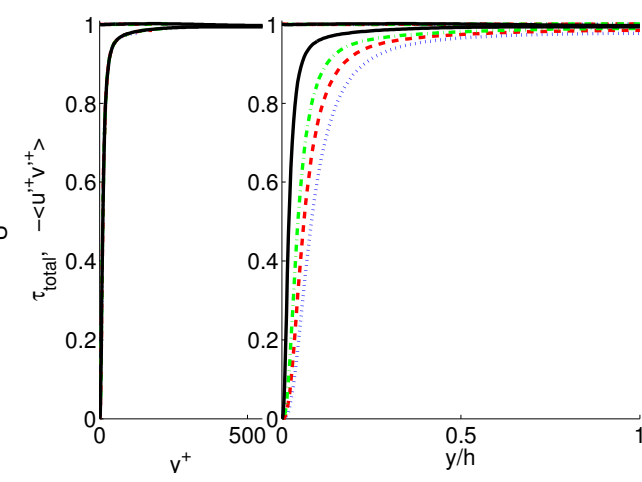

(b)

Figure 1: Color online. (a) Mean velocity profile scaled in outer (top-left, $U_{W}, h$ ) and inner (bottom-right, $u_{\tau},{ }^{+}$) scales. Lines as in Table 1. Circles from Tsukahara et al. (2006); squares from Bernardini et al. (2013). Blue thin solid line corresponds to viscous sublayer linear scaling law; red thin solid line represents near-wall classical logarithmic scaling law. (b) Total shear and Reynolds stresses in inner and outer scales.

$\begin{array}{llccccccccccc}\text { Case } & \text { Line } & R e_{\tau} & R e_{U_{w}} & L_{x} / h & L_{z} / h & \Delta x^{+} & \Delta z^{+} & N_{x} & N_{y} & N_{z} & u_{\tau} T / h & U_{c l} T / L_{x} \\ \mathrm{C} 125 & \cdots \cdots & 125 & 4500 & 20 \pi & 6 \pi & 13.7 & 6.18 & 864 & 151 & 576 & 27.1 & 7.4 \\ \mathrm{C} 180 & --- & 180 & 6300 & 20 \pi & 6 \pi & 13.1 & 6.6 & 1296 & 251 & 768 & 34 & 9.6 \\ \mathrm{C} 250 & - & 250 & 9000 & 20 \pi & 6 \pi & 10.2 & 6.1 & 2304 & 251 & 1152 & 53 & 15.5 \\ \mathrm{C} 550 & - & 550 & 22500 & 20 \pi & 6 \pi & 13.3 & 6.7 & 3888 & 251 & 2304 & 57 & 15.2\end{array}$

Table 1: Parameters of the simulations. $R e_{U_{w}}$ is the Reynolds number based on the velocity at the top wall and $h . L_{x}$ and $L_{z}$ are the periodic streamwise and spanwise dimensions of the numerical box, and $h$ is the channel half width. $\Delta x^{+}$and $\Delta z^{+}$are the resolutions in terms of Fourier modes. $N_{x}, N_{y}, N_{z}$ are the numbers of collocation points. The last two columns are the time span. Line shapes given in the second column have been used in figures $1 \mathrm{a} \mid 6$ and 8

indicator function is flatter for $R e_{\tau}=125,180$ and 250 than for $R e_{\tau}=550$ case and, in fact, it is much flatter than the one obtained for Poiseuille channel flow at $R e_{\tau}=550$. This may be an indication that in the Couette flow the slope constant $\kappa$ is less sensitive to the high Reynolds number effects than in the Poiseuille flow. Since with increasing Reynolds number log-region should become larger, the highest Reynolds number result shown on figure 2 may be not fully correct. We believe that the wavy behavior of the black thick solid curve is caused by the size of the box, which is large enough for the low Reynolds number simulations, but need to be investigated if is too small for $R e_{\tau}=550$ case.

An open question in Couette flows is the value of the non-dimensional velocity gradient at the channel centerline sometimes named slope parameter, defined as

$$
\Psi=\left.\frac{h}{U_{w}} \frac{\mathrm{d} U}{\mathrm{~d} y}\right|_{C L} .
$$

This value has been discussed in several works. Busse (1970), employing variational 


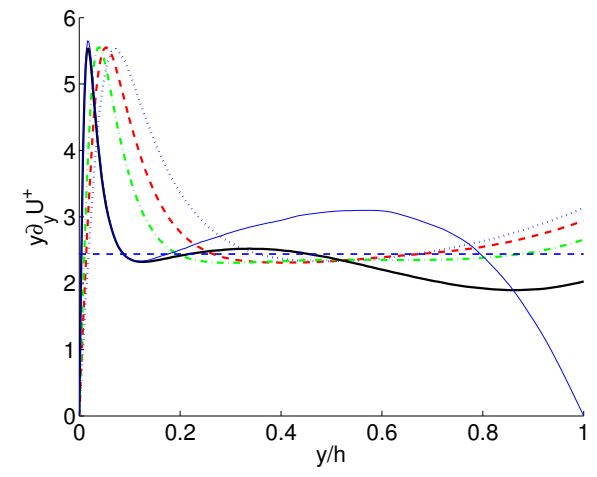

(a)

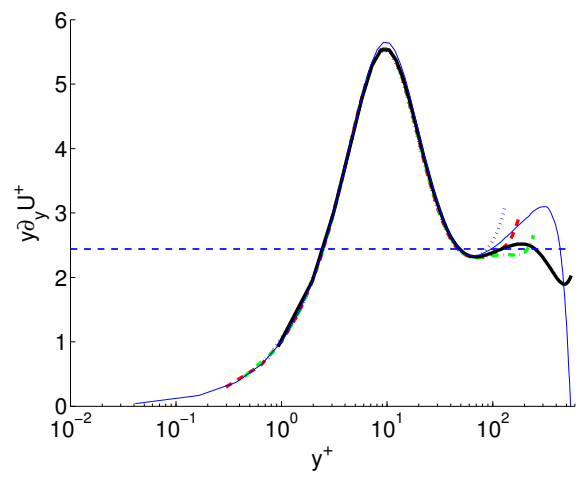

(b)

Figure 2: Color online. Log-indicator function, $(a, b)$ inverse Kármán constant in outer $(a)$ and wall $(b)$ units. The dashed straight line has a constant value of $1 / 0.41$. Lines as in Table 1. Blue thin solid line corresponds to the Poiseuille flow DNS at $R e_{\tau}=550$ (Jimenez \& Hoyas 2008).

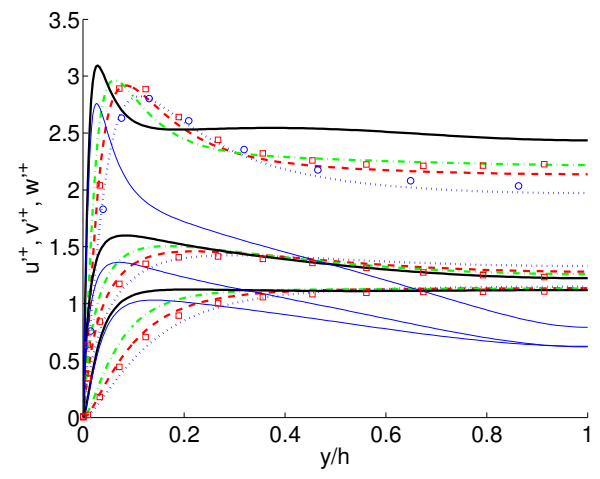

(a)

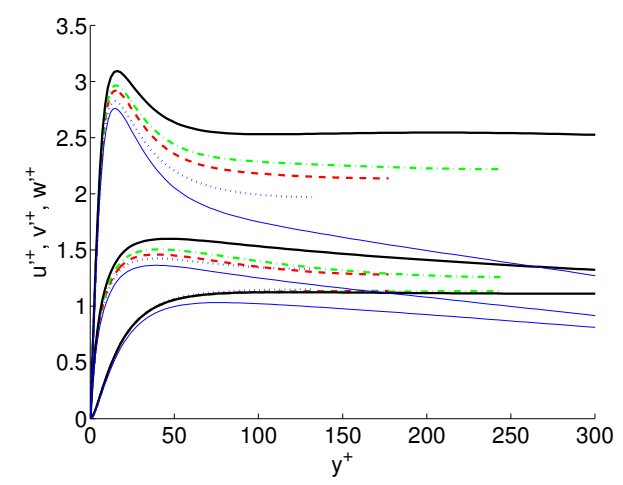

(b)

Figure 3: Color online. Velocity fluctuation intensities (rms) in outer $(a)$ and wall $(b)$ units. $u^{\prime+}, w^{\prime+}, v^{\prime+}$ from top to the bottom. Lines as in Table 1 Circles from Tsukahara et al. (2006); squares from Bernardini et al. (2013); thin blue lines, Poiseuille flow at $R e_{\tau}=550$ from del Álamo \& Jiménez (2003).

method and using experimental results of Reichardt (1959), determined a value of $\Psi=$ 0.25 at infinite Reynolds number, while Lund \& Bush (1980), performed an asymptotic analysis and concluded that it approaches zero as $R e \rightarrow \infty$. Several other DNS and experiments obtained values close to the range $0.18-0.2$, though all at low Reynolds numbers or very small boxes (Komminaho et al. 1996 , Tillmark 1995; Tsukahara et al. 2006). In our case a clear decrease is found for this parameter, with $\Psi=0.18,0.16,0.14$, and 0.1 for $R e_{\tau}=125,180,250$, and 550 reflected in figure 2a. Apparently this trend exhibits a rather slow convergence rate and it is inconclusive if in the limit $R e_{\tau}=\infty$ it will be zero or a small positive value.

The rms velocity fluctuation profiles are shown in figure 3 . Varying the Reynolds 


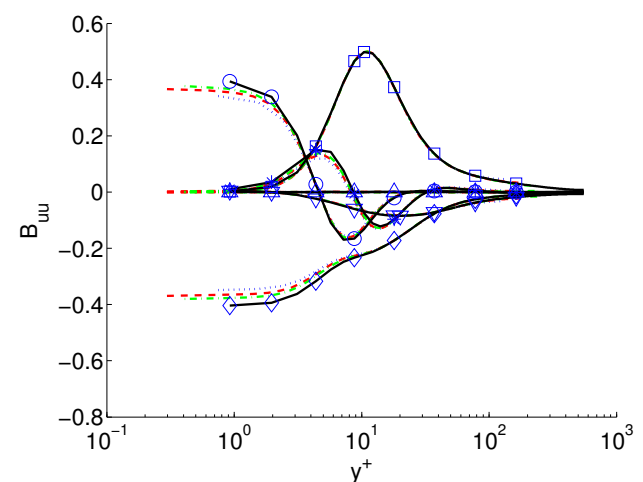

(a)

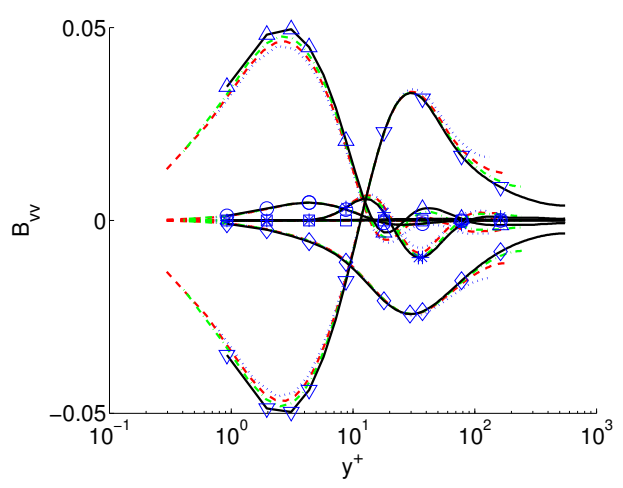

(c)

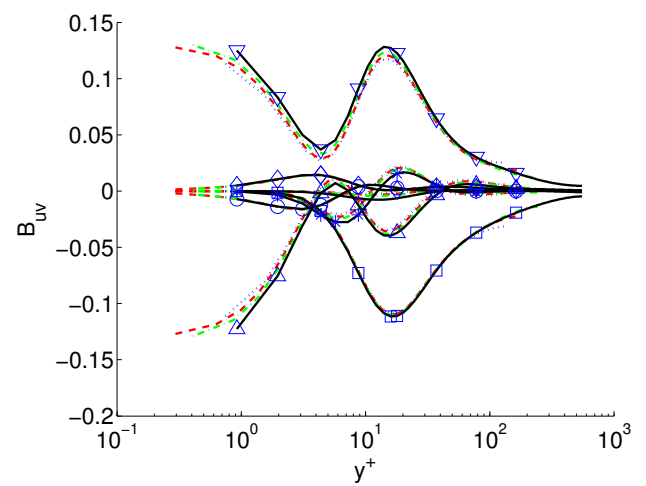

(b)

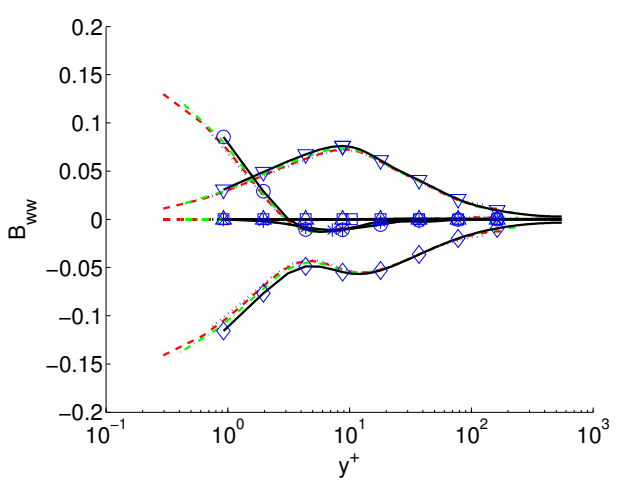

(d)

Figure 4: Color online. Budgets for the four nonzero Reynolds stresses. (a) $B_{u u} ;(b) B_{u v}$; (c) $B_{v v} ;(d) B_{w w}$. Production $\square$, dissipation $\nabla$, viscous diffusion $\circ$, pressure strain $\nabla$, pressure diffusion $\triangle$, turbulent diffusion $*$. Lines as in Table 1 .

number, neither $u^{\prime+}$ nor $w^{\prime+}$ collapses exactly in wall units. The peak of the $u^{\prime+}$ intensity at $15^{+}$is higher than the corresponding one in Poiseuille flows, as was also found in Bernardini et al. (2013). Thus, taking into account the result of Tsukahara et al. $(2006)$ that this value does not seem to be caused by the length of the box, we can conclude that even in the buffer layer, where this off-wall streamwise intensity peak is located, there is a difference between turbulent Couette and Poiseuille flows. Apart from this discrepancy in the value of the streamwise intensity maximum, the value of this peak follows the general tendency of wall bounded flows, increasing steadily with the Reynolds numbers. Away from the wall there is a clear tendency for $u^{\prime+}$ to increase with increasing Reynolds numbers, most noticeably at the center of the channel. This effect seems to be more important than the one in turbulent Poiseuille flows for the same range of Reynolds numbers, as was shown in Moser et al. (1999). In the case of $v^{\prime+}$ and $w^{\prime+}$ there is a less clear tendency to decrease. The almost perfect scaling of $v^{\prime+}$ both close and far away from the wall is remarkable. 


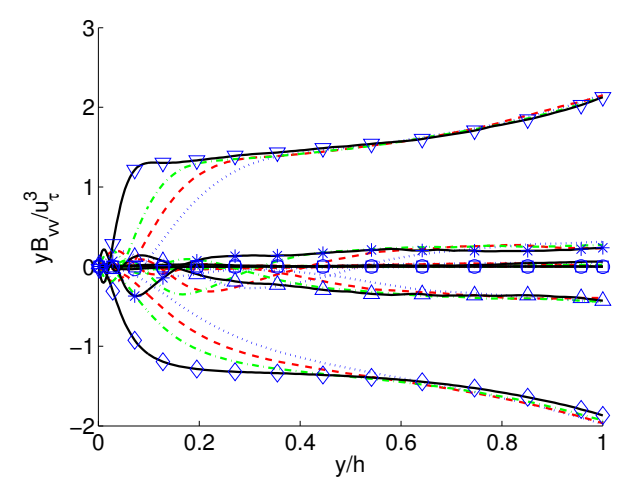

Figure 5: $B_{v v}$ adimensionalized by $u_{\tau}^{3} / h$ and premultiplied by the distance to the wall. Legends as in figure 4

\section{Turbulent budgets}

The budget equation for the component $\left\langle u_{i} u_{j}\right\rangle$ of the Reynolds-stress tensor, where $\left(u_{1}, u_{2}, u_{3}\right)=(u, v, w)$, is given by Mansour et al. (1988); Hoyas \& Jiménez (2008)

$$
B_{i j} \equiv \frac{D\left\langle u_{i} u_{j}\right\rangle}{D t}=P_{i j}+\varepsilon_{i j}+T_{i j}+\Pi_{i j}^{s}+\Pi_{i j}^{d}+V_{i j}
$$

where, $D / D t$ is the mean substantial derivative and \langle\rangle stands for averaging over the two homogeneous directions and time. The different terms in the right hand side are referred to as production, dissipation, turbulent diffusion, pressure-strain, pressure diffusion, and viscous diffusion. They are given as

$$
\begin{aligned}
P_{i j} & =\left\langle u_{i} u_{k}\right\rangle U_{j, k}-\left\langle u_{j} u_{k}\right\rangle U_{i, k}, \\
\varepsilon_{i j} & =-2 \nu\left\langle u_{i, k} u_{j, k}\right\rangle, \\
T_{i j} & =\left\langle u_{i} u_{j} u_{k}\right\rangle_{, k}, \\
\Pi_{i j}^{s} & =\left\langle p\left(u_{i, j}+u_{j, i}\right)\right\rangle, \\
\Pi_{i j}^{d} & =-\left[\left\langle p u_{i}\right\rangle \delta_{j k}+\left\langle p u_{j}\right\rangle \delta_{i k}\right]_{, k}, \\
V_{i j} & =\nu\left\langle u_{i} u_{j}\right\rangle_{, k k},
\end{aligned}
$$

where $\delta_{i j}$ is Kronecker's delta, the subscripts $(\cdot)_{, j}$ represent derivation with respect to $x_{j}$, and repeated subscripts imply summation over 1,2 and 3 . These budgets are shown in figure 4, non-dimensionalized by $\nu / u_{\tau}^{4}$. The maximum value of the residual is below $10^{-4}$ for all cases. As we can see the collapsing is perfect for $B_{u u}$ and $B_{w w}$ except for the dissipation and viscous diffusion. In the case of $B_{u v}$ and $B_{v v}$ the scaling is not perfect, mainly between 10 and 100 wall units. This is more remarkable in the pressure related terms, and it has been studied previously for Couette flows in Hoyas \& Jiménez (2008). The same conclusions obtained in that work applies here and are no repeated in sake of brevity. Note also the scaling failure near the centerline of $B_{v v}$, whose terms do not tend to zero there. This scaling failure is because the correct scale in the outer layer at a fixed distance to the wall is $u_{\tau}^{3} / h$. Figure 5 shows $B_{v v} / u_{\tau}^{3}$ premultiplied by the distance to the wall to show the $y^{-1}$ expected behavior of $B_{v v}($ Hoyas \& Jiménez 2008). Note that this behaviour holds relatively well up to $y=0.7 / h$, considerably longer than in Poiseuille flows. 


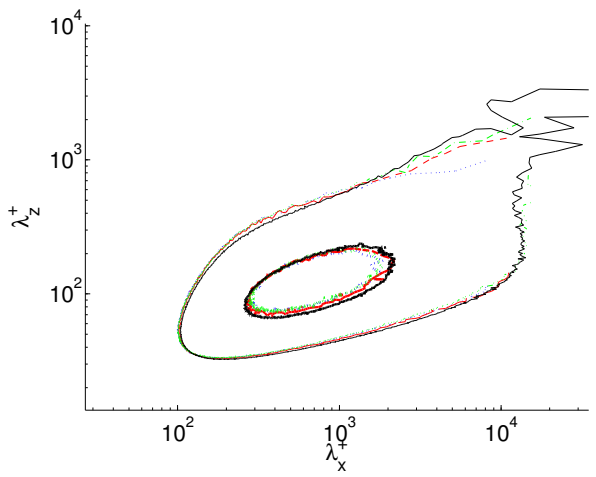

(a)

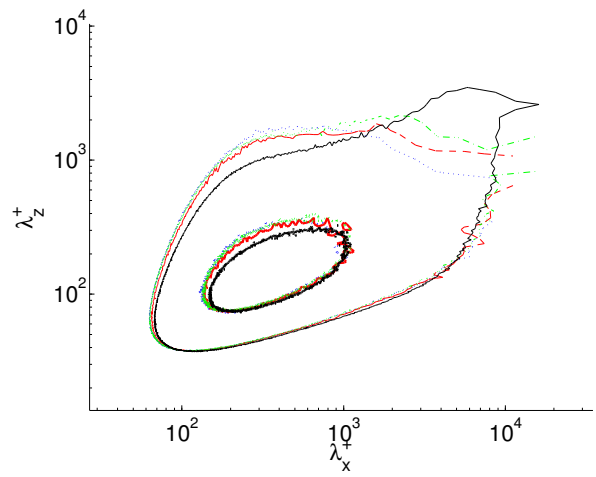

(b)

Figure 6: Color online. Spectral energy densities at $y^{+} \simeq 15$, in terms of the wavelengths $\lambda=2 \pi / k,(a) \phi_{u u},(b) \phi_{w w}$. Spectra are normalized in wall units, and the two contours for each spectrum are 0.125 and 0.625 times the maximum of the spectrum for the highest Reynolds number.

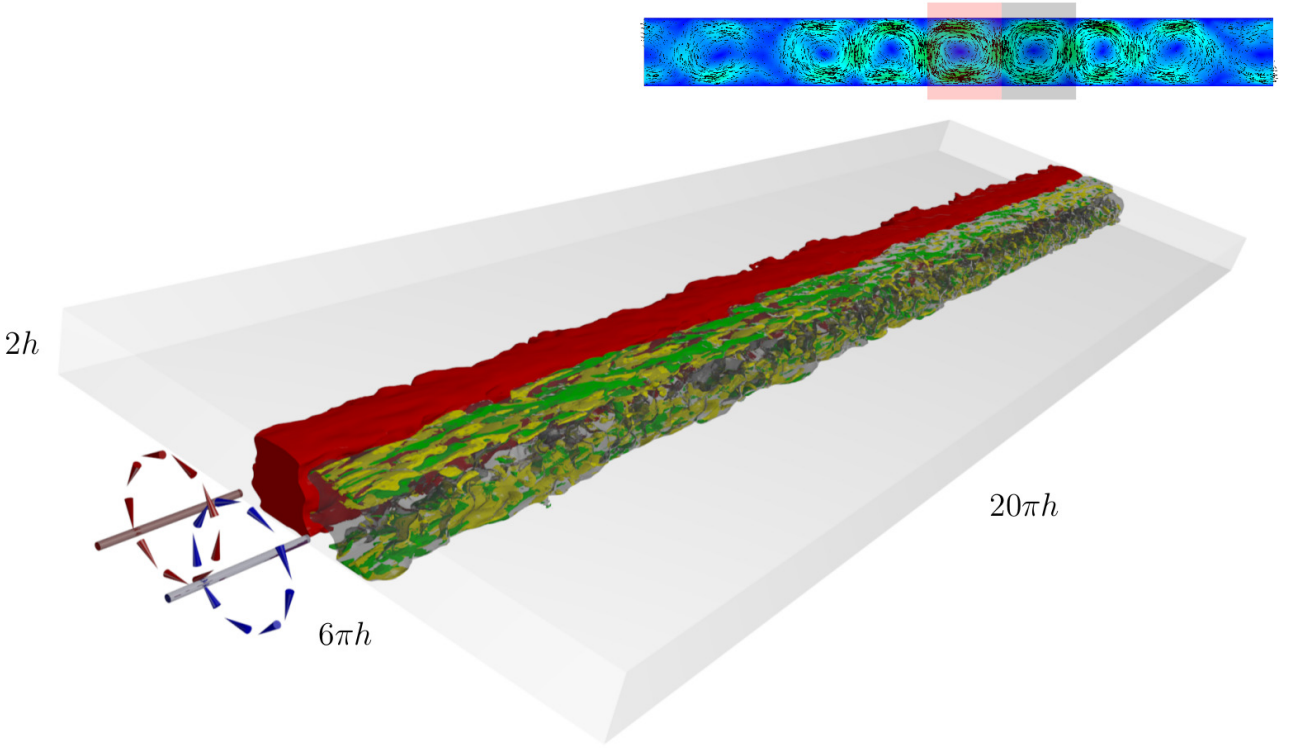

Figure 7: Color online. Coherent structures obtained from the ensemble average of the flow field spanning through all the channel and isosurfaces of $10 \%$ of the maximum vorticity in spanwise direction for $R e_{\tau}=125$.

\section{Structures}

In order to attain further structural information on the flow we consider two-dimensional spectral energy densities $\phi=k_{x} k_{z} E\left(k_{x}, k_{z}\right)$ at approximately $y^{+}=15$, the height of the near-wall kinetic-energy maximum, as shown in figure 6. As in Hoyas \& Jimenez (2006), two isolines are given for each case, representing the high-intensity core of the spectrum, 


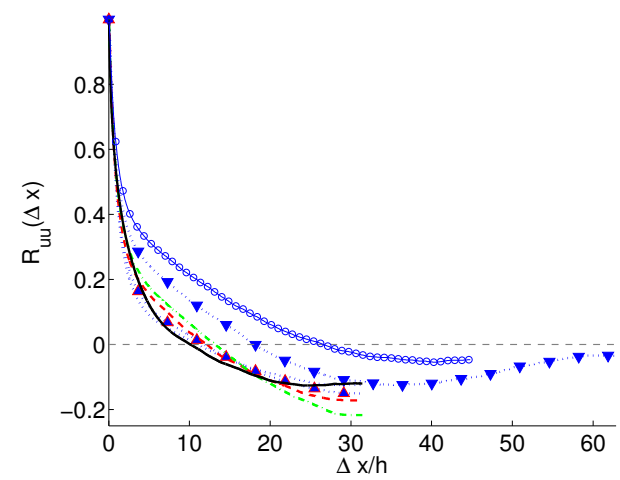

(a)

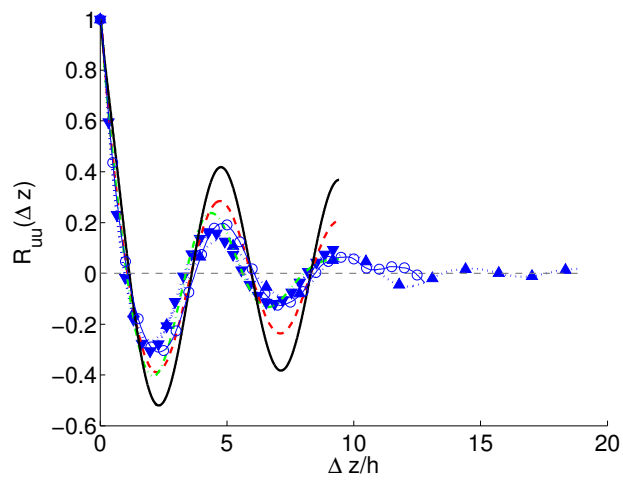

(b)

Figure 8: Color online. Two-point autocorrelation coefficient $R_{u u}$ of velocity fluctuations at the centerline, $y / h=0.5$. (a) Streamwise, (b) Spanwise. Circles from Tsukahara et al. (2006). $\triangle$ simulation for $R e_{\tau}=125,(20 \pi h \times 2 h \times 12 \pi h) ; \nabla, R e_{\tau}=125,(40 \pi h \times 2 h \times 6 \pi h)$.

and its outer border. As it happens in Poiseuille flows, the core isolines scale well in wall units, and the scaling failure appears for $u$ and $w$ in the upper right-hand corner, where a spectral ridge appears. The eddies in this ridge are inactive in the sense of Townsend (1976); Hoyas \& Jimenez (2006), as these structures do not appear in $v$ spectra or $u v$ cospectra. Far from the wall Couette and Poiseuille flows presents the same structure, but for the presence of a secondary peak in the Poiseuille flows spectra. This peak has been reported earlier (Tsukahara et al. 2006, Kitoh \& Umeki 2008) and corresponds to very long and wide turbulent patterns. These structures remain in the flow for a very long time, and their form can be recovered by an ensemble averaging in time, where $x-z$ plane averaging has been deactivated. The longest structures of the flow appear organized in counter-rotating pairs of rolls with high vorticity in their boundaries. An example is shown in figure 7 for $R e_{\tau}=125$, where we have isolated a pair of counterrotating rolls surrounded by iso-surfaces of vorticity in their boundaries. The position of these vortices in the channel is shown in the upper right corner of figure 7 . This subplot shows the velocity diagram of the mean in $x$ of the assembled average of ten turnovers of the $R e_{\tau}=125$ simulation. The presence of these footprints is a remarkable difference to Poiseuille flows, as in the latter they should be zero. Due to a quasi-periodic process (Hamilton et al. 1995) in the core region of the turbulent plane Couette flow low-speed velocity streaks generate large-scale vortices. The existence of such regeneration mechanism in the core region was confirmed experimentally by Kitoh \& Umeki (2008) and numerically by Pirozzoli et al. (2011) and Bernardini et al. $(2013)$. This process does not occur in a turbulent Poiseuille flows. Recently, Melnikov et al. (2014) investigated the stability of the long-wavelength instabilities and find that they are the result of the multiple small scale bifurcations that occur in the core region of the Couette flow.

These structures seems to be responsible for the long correlation length observed for Couette flows. The two-point autocorrelation coefficient $R_{u u}(\Delta z)$, is shown in figure $8 \mathrm{~b}$. The maxima are all at the same point for all Reynolds numbers studied when $L_{z}=6 \pi h$. In order to see if these structures are dependent of the box, we have made two more simulations for the $R e_{\tau}=125$ case, in boxes of size $(40 \pi h \times 2 h \times 6 \pi h)$ and $(20 \pi h \times 2 h \times$ $12 \pi h)$. The same structures and correlations appear in both geometries. Although it can 
be seen that the intensity of the autocorrelation $R_{u u}(\Delta z)$ is considerably reduced after $10 \Delta z / h$, it still present a clear sinusoidal pattern, with a difference between extrema of around $1.6 \pi h$, i.e., $0.8 \pi h$ per structure. This size agrees with the one given by other authors Tsukahara et al. (2006).

With reference to the length of these structures, Tsukahara et al. (2006) stated that up to $R e_{\tau}=126$ their length is $(40-65) h$, with a spanwise length of $(4-5) h$, later this result was confirmed by Kitoh \& Umeki (2008). In our simulations the autocorrelation coefficient $R_{u u}(\Delta x)$ does not present a secondary maximum below $60 h$, as it is shown in figure 8a. This result is obtained only for the lowest Reynolds number simulation $\left(R e_{\tau}=125\right)$ studied in the present paper. Thus, in order to capture the longest structures in the higher Reynolds numbers simulations the size of the box must be much larger.

\section{Conclusion}

We have presented a new set of turbulent plane Couette flow simulations. The main importance of the present paper lays in the investigation of the near-wall region of the flow. The computations at high Reynolds numbers $R e_{\tau}=180-550$ present an evidence of the presence of the logarithmic layer in turbulent Couette flow. It was found that the slope constant is equal to 0.41 and that the log-region in Couette flows is much longer than the one that can be found in turbulent Poiseuille flow at similar Reynolds numbers. Apart from that it was found that an additive constant $B$ does not depend on the Reynolds number and, most probably, this dependency, which was reported in Kitoh et al. (2005) and Tsukahara et al. (2006) is a low Reynolds number effect. Some of the other results found in the literature at lower Reynolds numbers are revised, such as the slope parameter $\Psi$ which is found to decrease to a small, though yet unknown limit. Differences and similitude with Poiseuille flows have been also revised. It is noteworthy to mention the failing in the scaling in wall units of the streamwise component of intensities, while their off-wall peak maximum is higher than in Poiseuille flows. As in the latter, the scaling failure also appears in the spectra of $u$ and $w$, the reason being that the eddies in this ridge are inactive in the sense of Townsend (1976); Hoyas \& Jimenez (2006). It was also found that away from the wall, most noticeably at the center of the channel, $u^{\prime+}$ is increasing with Reynolds number.

Far from the wall, long and wide structures, similar to those previously reported in the literature, and shown explicitly for the first time, are found. Their spanwise length does not depend on the size of the box. In order to decide the streamwise length of these structures, more simulations at very large boxes are needed.

This work was partially supported by the German Science Foundation (DFG) under the grant number KH 257/2-1 (2010). The computations of the new simulations were made possible by a generous grant of computer time from the FUCHS cluster at the University of Frankfurt-am-Main, the SuperMUC Petascale System at Leibniz Supercomputing Centre (LRZ) and the supercomputation center of the Universitat Politècnica de València. We are grateful to Messrs. Tsukuhara, Kawamura, Shingai, Bernardini, Pirozzoli and Orlandi for providing us with copies of their original data and to Mr. Pau Raga for his help in the preparation of figure 7. S. Hoyas is in debt with J. Jiménez.

\section{REFERENCES}

Del Álamo, J. C. \& JimÉnez, J. 2003 Spectra of the very large anisotropic scales in turbulent channels. Physics of Fluids 15 (6), L41-L44. 
Bech, K., Tillmark, N., Alfredsson, P. \& Andersson, H. 1995 An investigation of turbulent plane Couette flow at low Reynolds numbers. J. Fluid Mech 286, 291325.

Bernardini, M., Pirozzoli, S. \& Orlandi, P. 2013 The effect of large-scale turbulent structures on particle dispersion in wall-bounded flows. International Journal of Multiphase Flow 51, 55-64.

Busse, F.H. 1970 Bounds for turbulent shear flow. J. Fluid Mech 41, 219-240.

Hamilton, J.M., Kim, J. \& F., WalefFe 1995 Regeneration mechanisms of near-wall turbulence structures. J. Fluid Mech 287, 317-348.

Hoyas, S. \& Jimenez, J. 2006 Scaling of the velocity fluctuations in turbulent channels up to $R e_{\tau}=2003$. Physics of Fluids $\mathbf{1 8}(1), 011702$.

Hoyas, SERGio \& JimÉnEz, JaVier 2008 Reynolds number effects on the Reynolds-stress budgets in turbulent channels. Physics of Fluids 20 (10), 101511.

JimÉnez, J. 2013 Near-wall turbulence. Physics of Fluids 25 (10), 101302.

Jimenez, JAvier \& Hoyas, SERGio 2008 Turbulent fluctuations above the buffer layer of wallbounded flows. J, Fluid Mech. 611, 215-236.

Kim, J, Moin, P. \& R, Moser. 1987 Turbulence statistics in fully developed channels flows at low reynolds numbers. J. Fluid Mech 320, 259-285.

Kitoh, O., Nakabyashi, K. \& Nishimura, F. 2005 Experimental study on mean velocity and turbulence characteristics of plane Couette flow: Low-Reynolds-number effects and large longitudinal vortical structure. J, Fluid Mech. 539, 199-227.

Кітон, O. \& Uмекі, M. 2008 Experimental study on large-scale streak structure in the core region of turbulent plane Couette flow. Physics of Fluids 20 (2), 025107.

Komminaho, J., Lundbladh, A. \& Johansson, A. 1996 Very large structures in plane turbulent Couette flow. J, Fluid Mech. 320, 259-258.

LELE, S. K. 1992 Compact finite difference schemes with spectral-like resolution. Journal of Computational Physics 103 (1), 16-42.

Lund, K.O. \& Bush, W.B. 1980 Asymptotic analysis of plane turbulent Couette-Poiseuille flows. J. Fluid Mech 96, 81-104.

Mansour, N. N., Kim, J. \& Moin, P. 1988 Reynolds-stress and dissipation-rate budgets in a turbulent channel flow. Journal of Fluid Mechanics 194, 15.

Melnikov, K., Kreilos, T. \& B., Eckhardt 2014 Long-wavelength instability of coherent structures in plane couette flow. Phys. Rev. E 89, 043008-1-8.

Moser, R.D., Kim, J. \& Mansour, N.N. 1999 Direct numerical simulation of turbulent channel flow up to $R e_{\tau}=590$. Physics of Fluids 11 (4), 943-945.

Pirozzoli, S., Bernardini, M. \& Orlandi, P. 2011 Large-scale motions and inner/outer layer interactions in turbulent CouettePoiseuille flows. J. Fluid M 680, 534-563.

Reichardt, H. 1959 Gezetzmässigkeiten der geradlinigen turbulenten Couetteströmung. Mitteilungen aus dem Max-Planck-Institut für Strömungsforschung Göttingen 22.

Spalart, P. R. 1991 Spectral methods for the Navier-Stokes equations with one infinite and two periodic directions. Journal of Computational Physics 96 (2), 297-324.

Tillmark, N. 1995 Experiments on transition and turbulence in plane Couette flow. PhD thesis, KTH, Royal Institute of Technology.

Townsend, A.A. 1976 The Structure of Turbulent Shear Flows, 2nd ed.. Cambridge University Press, New York,.

Tsukahara, T., Kawamura, H. \& Shingai, K. 2006 DNS of turbulent Couette flow with emphasis on the large-scale structure in the core region. Journal of Turbulence 7, 1-16. 